\title{
Estudio de Satisfacción del Profesorado en la Universidad Pública Española
}

\author{
Rosario Frias AZCÁrate \\ Universidad de Alcalá \\ rosario.frias@uah.es
}

Recibido: 7.03.2006

Aceptado: 1.06 .2006

\section{INTRODUCCIÓN}

En los inicios del siglo XXI la Universidad Española se encuentra ante el reto de dar respuesta a las necesidades y demandas que están configurando una nueva sociedad. Esta nueva realidad social, que se ha denominado sociedad en red (Castells, 1999), afecta ineludiblemente a la institución universitaria.

Los rasgos básicos que la definen están delimitados por dos características fundamentales: el imparable proceso de globalización que acerca e interrelaciona a los distintos países y comunidades del mundo, generando simultáneamente proximidad y competitividad, y el incremento de autonomía que demandan cada vez más comunidades y áreas culturales integradas en los distintos países.

En este sentido, las instituciones universitarias se ven afectadas por la necesidad de ser cada día más universales y dotadas de un capital humano y técnico más moderno, actualizado y competitivo, a la vez que deben dar respuesta a la realidad social inmediata en la zona de circunscripción a la que pertenecen. Por ello, hay un doble movimiento simultáneo en el mundo universitario: la existencia de mayor interrelación y de mayor autonomía.

La Universidad Española ha incorporado como valores dominantes de referencia en su horizonte de desarrollo, la calidad y la excelencia. Dichos valores han de plasmarse en realidades fácticas, por lo que son objetivos prioritarios en los proyectos de corto, medio y largo plazo en el ámbito universitario.

Pero hablar de calidad y de excelencia supone todo un conjunto de aspectos que hay que afrontar. De hecho, ya se han formalizado indicadores que permiten evaluar estos conceptos para las distintas universidades españolas (de Miguel et al., 2001). La consecución de niveles óptimos de satisfacción del profesorado universitario es uno de los aspectos que ineludiblemente incide de manera directa en la realización de políticas encaminadas a la materialización de estos conceptos. Es obvio que la satisfacción de estos profesores repercute en el buen funcionamiento de la universidad en su conjunto, así como, la aplicación de estra- 
tegias idóneas revierte en la satisfacción de los mismos. De manera que se produce un bucle doble, cuyo resultado es un proceso de retroalimentación entre ellos.

Por otra parte, la Universidad Pública Española (en adelante UPE) está inmersa en estos momentos en importantes procesos de cambio como consecuencia de la aplicación y modificación de la Ley Orgánica de Universidades (en adelante LOU) tras su aprobación en el año 2001. Asimismo, tiene en el horizonte la realización de los procesos de convergencia basados en los acuerdos de Bolonia.

Por tanto, la UPE, se halla en un momento de transformaciones que afectan prácticamente a la totalidad de los elementos que la constituyen.

El resultado de todo este proceso ha de ir encaminado a dotar a esta Institución de mayores niveles de calidad y excelencia. De esta manera, será posible incrementar su capacidad competitiva con otras universidades europeas, y también extraeuropeas, para afrontar con garantías el futuro inmediato de este mundo globalizado en el que nos encontramos.

Por todo ello, resulta de vital importancia: a) que el desarrollo y los cambios de la LOU incidan en la dirección apuntada anteriormente, de forma que dicha Universidad pueda ofrecer unos conocimientos dentro de los parámetros de calidad de las universidades europeas punteras. b) que se realice una investigación dentro de esos mismos criterios. C) que su Personal Docente e Investigador (en adelante PDI) reúna los requisitos adecuados y a su vez se les dote de los elementos necesarios para llevar a cabo su función de forma satisfactoria.

De lo que suceda en estos próximos años cruciales, en cuanto a: a) la configuración que adopte el modelo universitario público español, b) los medios técnicos y humanos de que disponga, c) los criterios que imperen acerca del perfil del PDI, d) los métodos de selección de este colectivo, e) su itinerario profesional en el seno de la institución, f) las políticas de promoción e incentivación laboral y profesional que se consoliden, g) el nivel y tipo de relación con otras universidades y con otras instituciones y organizaciones sociales, dependerá el tipo de universidad pública que tengamos en un futuro inmediato en nuestro país y, por tanto, de su capacidad competitiva con relación a las universidades de mayor prestigio.

En el diseño del proyecto se ha tenido presente la existencia de numerosos estudios sobre satisfacción y sobre formalización de indicadores que permiten evaluarla. Sin embargo, son escasas las investigaciones que permitan conocer la "satisfacción" de este colectivo, desde lo que él mismo considera, define y jerarquiza.

Abordar el análisis de la satisfacción del profesorado universitario desde este enfoque permite conocer cuáles son los ámbitos de significación y de sentido para este colectivo y, por tanto, establecer criterios de evaluación desde su propia experiencia. Todo ello redundará en unos resultados que se contextualizarán en un plano más próximo a la realidad y traerá como consecuencia una posibilidad de aplicación más fecunda para establecer políticas y estrategias de mejora en este campo. 
En primer lugar, se ha querido conocer en profundidad el grado de satisfacción laboral que tienen los profesores de la UPE a partir del conocimiento de lo que para ellos significa y representa este concepto. Por tanto los propios profesores definen los criterios, sin apriorismos que condicionen y delimiten el campo de información obtenida.

Para lograr ese tipo de información hemos considerado que la opción metodológica pertinente era el enfoque cualitativo: el grupo de discusión y la entrevista en profundidad. Dado que se trata de desvelar aquellos aspectos estructurales existentes, más allá de lo directamente observable y cuyo funcionamiento constituye la logica profunda, es decir, el funcionamiento subyacente a partir del cual se explica el orden aparente.

Se ha utilizado:

La entrevista en profundidad: para los casos que poseen un conocimiento, una implicación y una posición específica respecto al tema objeto de estudio.

Las reuniones de grupo: con el fin de reproducir situaciones en las que se den relaciones, interconexiones, interdependencias, reciprocidad entre sus miembros, semejantes a las que se producen en la realidad. Las reuniones de grupo aportan la posibilidad de alternar fases de discurso racional con fases de tipo proyectivo, en las cuales, a través de técnicas específicas, pueden manifestarse las motivaciones más profundas. Además, el grupo, por su propia dinámica es un dispositivo que facilita la expresión y producción de ideas, tanto en su contenido latente como en su contenido manifiesto.

Por tratarse de una investigación cualitativa se ha trabajado sobre una muestra estructuralmente representativa de la realidad estudiada. Los entrevistados y los componentes de las reuniones de grupo son seleccionados bajo el criterio de «pertinencia» como criterio de representatividad de las estructuras y relaciones relevantes, en la que cada unidad de la muestra se encuentra relacionada con todas las demás.

Las variables que se han contemplado para la formalización de la muestra son las siguientes:

- Localización geográfica: Madrid, Barcelona. Se ha optado por la selección de Madrid y Cataluña como dos grandes comunidades de referencia, por tratarse de comunidades dotadas de Universidades tanto Central como Autónoma como Politécnica.

- Género: con el fin de poder detectar si se produce alguna diferencia respecto a la satisfacción según se trate de profesores o profesoras. Esta variable, puede estar asociada al área de conocimiento.

- Área de conocimiento: Se tendrán en cuenta las cuatro áreas de conocimiento fundamentales. CC. Experimentales y de la Salud, CC. Sociales y Jurídicas, Humanidades y Enseñanzas Técnicas. Para contemplar todas las áreas de conocimiento se realiza el trabajo de campo en las Universidades de Madrid (Complutense, Autónoma y Politécnica) y de Barcelona (Central, Autónoma y Politécnica). 
- Tipo de profesorado: fijo o contratado. Esta diferencia respecto al tipo de vinculación contractual con la institución universitaria es previsible que tenga una repercusión relevante en la experiencia de la satisfacción

Por tato la muestra queda constituida de la siguiente forma:

\begin{tabular}{|c|c|c|c|}
\hline $\begin{array}{c}\text { UNIVERSIDAD } \\
\text { CIUDAD }\end{array}$ & $\begin{array}{c}\text { U. } \\
\text { CENTRAL }\end{array}$ & $\begin{array}{c}\text { U. } \\
\text { AUTÓNOMA }\end{array}$ & $\begin{array}{c}\text { U. } \\
\text { POLITÉCNICA }\end{array}$ \\
\hline MADRID & $1 \mathrm{G}$ & $1 \mathrm{G}$ & $1 \mathrm{G}$ \\
\hline BARCELONA & $1 \mathrm{G}$ & $1 \mathrm{G}$ & $1 \mathrm{G}$ \\
\hline
\end{tabular}

Total 6 Grupos

\begin{tabular}{|c|c|c|c|c|}
\hline & \multicolumn{2}{|c|}{$\begin{array}{c}\text { PROFESORES } \\
\text { FIJOS }\end{array}$} & \multicolumn{2}{|c|}{$\begin{array}{c}\text { PROFESORES } \\
\text { CONTRATADOS } \\
\end{array}$} \\
\hline & MADRID & BARCELONA & MADRID & BARCELONA \\
\hline UC & 3 & 3 & 3 & 3 \\
\hline CC. exp. y salud & $1 \mathrm{H}$ & $1 \mathrm{M}$ & $1 \mathrm{M}$ & $1 \mathrm{H}$ \\
\hline CC.sociales y jur. & $1 \mathrm{H}$ & $1 \mathrm{M}$ & $1 \mathrm{M}$ & $1 \mathrm{H}$ \\
\hline Humanidades & $1 \mathrm{M}$ & $1 \mathrm{H}$ & $1 \mathrm{H}$ & $1 \mathrm{M}$ \\
\hline UAM & 3 & 3 & 3 & $\mathbf{3}$ \\
\hline Cc. exp. Y salud & $1 \mathrm{M}$ & $1 \mathrm{H}$ & $1 \mathrm{H}$ & $1 \mathrm{M}$ \\
\hline Cc. sociales y jur. & $1 \mathrm{M}$ & $1 \mathrm{H}$ & $1 \mathrm{H}$ & $1 \mathrm{M}$ \\
\hline Humanidades & $1 \mathrm{H}$ & $1 \mathrm{M}$ & $1 \mathrm{M}$ & $1 \mathrm{H}$ \\
\hline UP & 1 & 1 & 1 & 1 \\
\hline E. Técnicas & $1 \mathrm{H}$ & $1 \mathrm{M}$ & $1 \mathrm{M}$ & $1 \mathrm{H}$ \\
\hline
\end{tabular}

Total 58 Entrevistas

A través de estas técnicas se pretende conocer las motivaciones más profundas (causas, deseos y necesidades de las actitudes expresadas) que conducen a los profesores de la UPE a desempeñar su trabajo; las actitudes (pautas más o menos estables de actuación y comportamiento en el trabajo), las creencias y valores en los que se sustentan dichas motivaciones y actitudes, desvelando mediante su conocimiento la situación de satisfacción/insatisfacción que estos profesionales experimentan en su trabajo (Frías, 1994). 
La realización de grupos de discusión se ha llevado a cabo siguiendo la técnica desarrollada en la obra clásica de Jesús Ibáñez (1979). Las entrevistas en profundidad se basan en el enfoque que aporta Alfonso Ortí en el texto sobre la entrevista individual abierta semidirectiva (Ortí, 1989). Se han realizado seis grupos de discusión y veintiocho entrevistas en profundidad, teniendo en cuenta las variables: localización geográfica (Madrid, Barcelona), género (profesores, profesoras), área de conocimiento (ciencias experimentales y salud, ciencias jurídicas y sociales, humanidades y enseñanzas técnicas) y tipo de relación contractual con la institución (fijo, contratado).

El enfoque elegido para el análisis de los discursos producidos por los grupos ha sido el semiótico-estructural desde una perspectiva sociológica, atendiendo a la semiótica del relato y a los análisis estructurales de Lévi-Straus (1977), Greimas (1973), Barthes (1971) y Bourdieu (1985).

Esta metodología ha resultado idónea porque ha permitido profundizar y conocer aspectos que no hubiesen sido posibles utilizando otra herramienta metodológica. Además, ha aportado una cantidad y calidad de información como consecuencia de la creación de escenarios donde los profesores hablaban con plena libertad y sin imposición de planteamientos directivos.

Por tanto, este estudio pretende ser un instrumento de apoyo para incrementar la satisfacción en el trabajo de los profesores universitarios, lo que redundará, en la presencia de una Universidad Pública de mayor calidad.

\section{CONTEXTUALIZACIÓN}

La producción discursiva de los profesores sobre su trabajo, sus narraciones y valoraciones, se sustentan en buena medida en el entrecruzamiento de varios planos que atraviesa de forma casi permanente sus discursos. Esto es inherente a cualquier proceso discursivo generado de modo no directivo. En este caso es importante señalarlo por el grado de significatividad que adquiere, para una mejor comprensión de estos procesos en los profesores universitarios.

Es necesario considerar que en ocasiones sus comentarios y valoraciones no se hacen de manera plenamente consciente, lo que el análisis evidenciará nítidamente. Esto adquiere una relevancia fundamental para la configuración del marco analítico-explicativo.

La posición cognitivo-valorativa que mantienen sobre su mundo laboral viene predeterminada por la referencia a la Universidad desde dos perspectivas: como institución concreta (plano de lo real) y como imaginario individual y colectivo (plano de lo simbólico). Ambos ámbitos están presentes y se mezclan de forma indiferenciada en los profesores, de forma tal que la Universidad es a la vez, una institución en la que trabajan y un desideratum que comporta la adscripción y la investidura del sujeto, mediante un proceso osmótico, de los atributos y valores social y culturalmente establecidos, asociados al mundo universitario (plano de lo ideal, del "deber ser").

EMPIRIA. Revista de Metodología de Ciencias Sociales. N. ${ }^{\circ} 11$, enero-junio, 2006, pp. 175-201. ISSN: $1139-5737$ 
Para el profesor universitario lo laboral se relaciona con:

- El mundo de lo concreto: aspectos materiales y humanos, tanto coyunturales como estructurales.

- El mundo de los intangibles: atributos, valores, símbolos, etc. Todo ello constituido, legitimado y alimentado desde el imaginario colectivo.

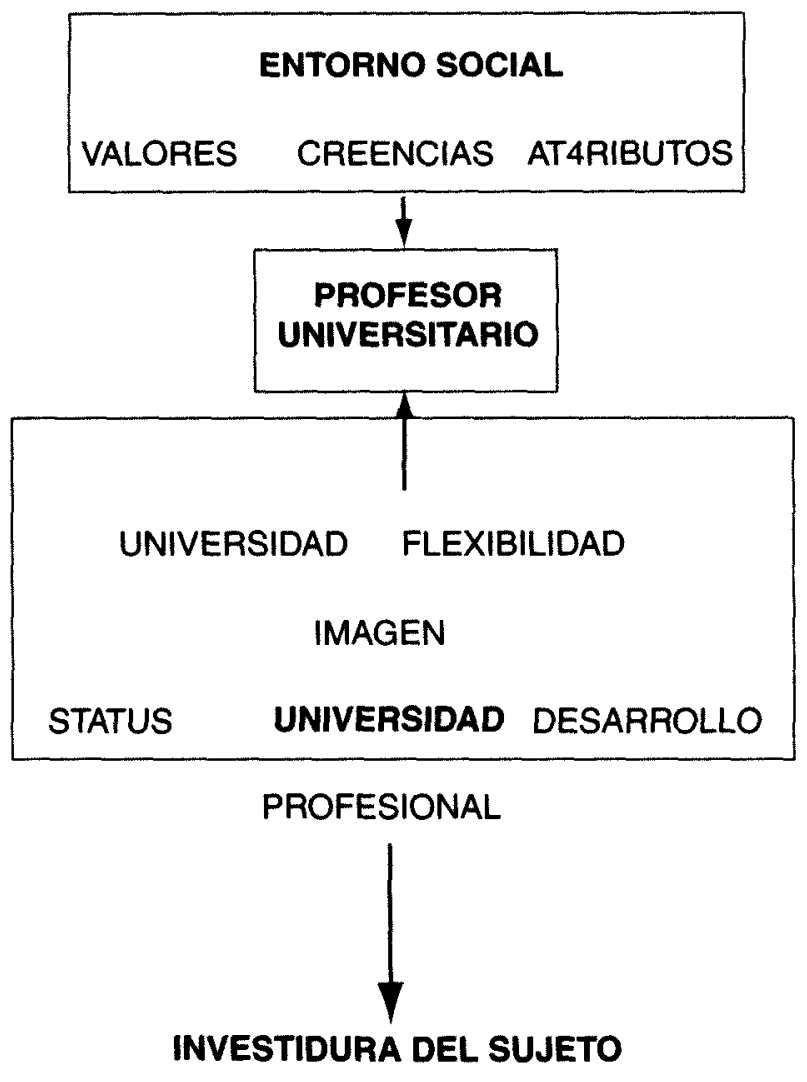

Fig. 1: Significado del trabajo para el profesor universitario

Ambos planos se sitúan en la Institución Universitaria donde, sobre todo el segundo, configuran en buena medida el proceso narrativo y el diagnóstico de su situación laboral (véase Fig.1). Sin embargo, hay que matizar que existen diferencias significativas en relación con el área de conocimiento a la que pertenecen los profesores. Quienes pertenecen al ámbito de las escuelas y facultades técnicas tienden a hacer mayor hincapié en los aspectos concretos, dejando de lado aquellos que se refieren al plano de lo simbólico, mientras que para el resto de los profesores están presentes ambos planos. 
Otro elemento importante es la omisión del término trabajo. Apenas aparece cuando se les pide que hablen de su situacion laboral. Este hecho obedece a un doble motivo: a) la conciencia de estar realizando una labor profesional diferente y especial, que difícilmente asocian e identifican como trabajo, y b) la autoimagen de ser profesores universitarios.

El término trabajo tiene connotaciones negativas y se asocia con cuestiones como: horario que hay que cumplir, control sobre las tareas que se realizan por parte de la figura de un jefe, dedicación a algo que genera un sobreesfuerzo porque no gusta, etc. Además, quien realiza un trabajo es un trabajador. Por tanto, los profesores apenas hacen uso del término porque se perciben y valoran como profesionales, que disponen de flexibilidad y de libertad para la realización de sus funciones, sienten que carecen de la figura de un jefe y que, además, realizan una labor profesional que les gusta.

Otra faceta que hay que remarcar, es que el objeto de análisis no es ajeno al contexto historico, social y cultural de nuestro país. Las opiniones y valoraciones que realizan se han de enmarcar y relacionar con los estados de opinión de nuestra sociedad para poder generar una explicación de mayor profundidad. Por tanto, los discursos de los profesores se interpretan simultáneamente como producción y efecto de sentido. Es decir, los sujetos hablan desde posiciones personales, que son generadoras de sentido; pero, a su vez, lo hacen reproduciendo fragmentos de discursos que circulan socialmente, que son el reflejo de estados de opinión dominantes. Todo esto queda patente en el conjunto de los profesores. Tan sólo sirva como referencia, de lo que más adelante se hará explícito: lo más repetido en relación con la satisfacción laboral es "la búsqueda de reconocimiento institucional y social". No es casual la alusión tan frecuente a lo social como referente de su situación laboral. Es decir, el entorno social opera como un importante referente de la Institución Universitaria desde el punto de vista de la valoración que existe sobre la misma y, por tanto, de la imagen que sienten que hay en la sociedad sobre su profesorado.

Los profesores hablan fundamentalmente como sujetos que están dentro del sistema universitario. Desde este espacio en el que se sitúan, sienten y perciben el ámbito social como entorno, pero en determinados momentos, minoritarios, de las entrevistas y grupos de discusión también se posicionan como individuos que están inmersos en la sociedad a la que pertenecen. Este desdoblamiento de planos de pertenencia evidencia la enorme fuerza que adquiere la adscripción institucional, ya que cuando se sitúan en el ámbito universitario existe un discurso fuertemente defensivo hacia lo social al instalarse "la amenaza del mundo exterior" como evaluador, con capacidad para "reconocer" o no, tanto las tareas propias del profesor universitario como el status y prestigio que consideran que conlleva. Cuando, se posicionan como miembros de la sociedad, su discurso tiende a ser más "relajado", más analítico y, más objetivo.

El mundo laboral de los profesores universitarios se percibe con unas características específicas, muy distintas de otros ámbitos laborales, no sólo en comparación con el área de las empresas privadas sino también con respecto a 
otras instituciones públicas. De hecho, lo laboral no representa un espacio concreto ni un tiempo definido; más bien, se sitúa en un territorio que lo trasciende, que se define por parámetros que tienen que ver con criterios asociados a "opciones de vida", "estilos de vida", etc. Esto significa, a su vez, que tampoco se sitúa solamente en la esfera de lo público, como es dominante en otros ámbitos de trabajo, sino que lo público y lo privado se entremezclan. A los profesores universitarios les resulta difícil diferenciarse desde el nivel de lo consciente, y en un plano preconsciente o inconsciente el discurso pone de manifiesto el solapamiento de ambos.

Por tanto, lo que aquí se quiere mostrar, como una primera aproximación, es el marco que representa el mundo laboral y los aspectos que ayudan a contextualizar este marco, cuyo elemento más definitorio es que contiene características muy específicas que hacen que resulte bien diferente de otros, según manifiestan los discursos de estos profesores. $\mathrm{Y}$ es necesario resaltar que esas diferencias y especificidades hay que situarlas tanto en el plano de lo real, lo concreto/material, como en el plano simbólico, del imaginario de los sujetos.

En definitiva, los profesores universitarios cuando hablan acerca de su escenario laboral lo hacen relacionándolo tanto con el desempeño de sus funciones y tareas, como con la Institución en y para la que trabajan. Ambos planos están cargados de unos atributos bien diferentes de otros ámbitos laborales, que poseen una fuerza tal que llegan a constituir elementos fundamentales en su identidad como sujetos. De manera que de la carrera académica, lo que valoran como éxitos o fracasos, no afecta sólo a lo estrictamente profesional sino que empapa a aspectos que tienen que ver con la autopercepción y autovaloración como sujetos.

\section{PERCEPCIÓN Y ANÁLISIS DE LA SITUACIÓN LABORAL DE LOS PROFESORES UNIVERSITARIOS: ASPECTOS POSITIVOS $Y$ NEGATIVOS}

La valoración que realizan sobre su situación laboral viene determinada fundamentalmente por el tipo de relación contractual que tienen con la Universidad. Existe una rotunda quiebra discursiva entre los profesores fijos y los contratados. De manera que esta variable es la más discriminante del conjunto de las que han constituido la muestra.

Esta ruptura se traduce en:

- Una posición más crítica por parte de los profesores contratados al valorar su situación laboral.

- Una posición menos crítica por parte de los profesores fijos, que se refleja en el grado de intensidad de los aspectos enunciados como negativos y en una posición más tendente a la autocomplacencia. 
Ahora bien, existe una posición unánime al evaluar la situación laboral, destacando que los elementos negativos son muy superiores cuantitativa y cualitativamente a los positivos.

En mayoría de investigaciones que se realizan sobre satisfacción laboral se produce una tendencia inercial a remarcar los puntos más negativos en detrimento de los positivos. Ello se ha tenido en cuenta en este estudio, y por tanto en el análisis de los resultados. Sin embargo, hay que poner un énfasis especial en las razones concretas que permiten entender este posicionamiento claramente crítico, mostrar sus hechos diferenciales específicos y, en definitiva, conocer los ejes estructurales sobre los que se asienta una posición tan aplastantemente mayoritaria sobre este tema.

La otra variable que ha tenido un papel discriminante ha sido el área de conocimiento. Se ha constatado que existen unos planteamientos diferentes sobre algunos temas entre los sujetos adscritos a las escuelas y facultades técnicas y el resto de la muestra. Las razones básicas de estas diferencias se encuentran en que las Escuelas y Facultades Politécnicas presentan situaciones específicas que no existen en el resto, siendo las principales las siguientes:

- Poseen una situación económica mejor, debido fundamentalmente a la obtención de proyectos externos a la Universidad, ya sean de instituciones públicas como de contratos con empresas privadas. La cuantía económica de estos proyectos suele ser muy superior al resto de las Universidades analizadas y con una clara perspectiva de aplicación de los resultados obtenidos.

- Los profesores de las Escuelas y Facultades Politécnicas poseen una cultura institucional diferente del resto de la muestra. En este sentido, existen unos anclajes históricos, que tienen que ver tanto con el sistema organizativo como con el desempeño de funciones y con los valores instalados en los profesores, que generan algunas posiciones diferenciales con el resto de las Facultades. Por ejemplo, la importancia que tiene hoy la figura de catedrático en la politécnica, un poder tanto material como simbólico, es muy superior al de las otras Facultades. Tienden a generar un discurso más pragmático. Sus opiniones y valoraciones se sitúan más claramente en aspectos concretos y son menos propensos a ejercer posiciones críticas desde el mundo de lo simbólico. Es decir, estos profesores tienen un discurso más apegado al plano de lo real, tanto en sus valoraciones positivas como negativas; $y$, por el contrario, el plano de lo simbólico no emerge con la misma intensidad que en el resto de los profesores de otras áreas.

El resto de las variables que han configurado la muestra no presentan diferencias significativas desde un análisis global, aunque sí aparecen matices que rompen la homogeneidad en aspectos muy concretos.

Como se señalaba más arriba hay una postura prácticamente unánime sobre la valoración predominantemente negativa de su situación laboral. Se produce un 
hecho profundamente significativo y que no pertenece al material analizado: la dificultad de reclutamiento de la muestra como consecuencia de las resistencias a hablar de su situación. Este hecho permite entender con mayor profundidad algunos aspectos sobre la situación existente y sobre las opiniones y valoraciones que de esta situación realizan los entrevistados. Refleja un sinfín de elementos que están interviniendo actualmente. Sin embargo, se destacan tres interrelacionados entre sí y que guardan una total coherencia con lo analizado en los discursos de los profesores:

a) La desconfianza como actitud generalizada.

b) La falta de credibilidad en la traducción operativa de los resultados.

c) La competitividad, en su vertiente peyorativa, como criterio dominante de este colectivo.

Este discurso de la queja, consolidado en el conjunto de los profesores entrevistados, adquiere distintos matices e intensidades, como ya se señaló, según provenga de profesores fijos o de profesores contratados. Esta variable transmite la línea de demarcación básica, entre posiciones que son unánimes, pero que se estructuran con distintos matices y con diferente intensidad. Ello obliga a estructurar la información de los aspectos positivos y negativos en función de dicha variable.

Los aspectos negativos más relevantes para los profesores contratados son los siguientes:

- En relación a su situación laboral:

- precariedad laboral.

- En relación a la carrera académica:

- incertidumbre sobre su futuro profesional.

- falta de una planificación clara y precisa de la carrera académica.

- posición de vulnerabilidad, debido a la situación de dependencia de los profesores fijos, principalmente, de los catedráticos de universidad.

- ausencia de una perspectiva de futuro profesional dentro de la Universidad.

- En relación a la institución:

- falta de adecuación entre las obligaciones formales en el desempeño de sus tareas, las funciones que se reflejan en su contrato y las obligaciones reales que llevan a cabo.

- despachos compartidos por muchos profesores y en malas condiciones físicas y ambientales.

- escasez de recursos y medios económicos y técnicos para la realización de sus tareas docentes e investigadoras.

- imposibilidad de tener acceso a proyectos de investigación.

- falta de canales formales de información y comunicación con la Institución Universitaria: falta de transparencia. 
- falta de reconocimiento institucional y social de su trabajo.

- masificación de las aulas en los primeros cursos de carrera.

- características del alumnado: mayoritariamente pasivo y con bajo nivel de formación.

Los aspectos negativos más relevantes para los profesores fijos son los siguientes:

- En relación a su situación laboral:

- consideración que poseen salarios bajos, no ya sólo en relación con el mundo de las empresas privadas sino en comparación con otros cuerpos de funcionarios de categoría similar.

- En relación a la carrera académica:

- absoluta prioridad existente de la investigación como único criterio curricular, y más que de la investigación de la publicación, en detrimento de la docencia.

- En relación a la institución:

- tener que verse obligados a realizar tareas que no les corresponde: gestión burocrático-administrativa vinculada a la investigación.

- ausencia de reconocimiento en las funciones de gestión.

- malas condiciones de los despachos y, en ocasiones, el excesivo número de personas que lo comparten

- falta de medios económicos, técnicos y humanos para la realización de su trabajo. Algunos consideran que no se trata tanto de una falta de medios económicos, sino de una mala gestión de los recursos disponibles. Las diferencias valorativas entre unos y otros no se relacionan con las variables que segmentan la muestra, más bien obedecen a criterios personales.

- fuerte incremento de posiciones individualistas en el seno de los departamentos, frente a posturas más solidarias y de compañerismo en otras épocas.

- falta de transparencia en los flujos de información en todos los niveles de la Institución.

- falta de reconocimiento institucional y social.

- sensación de estar desasistidos por parte de la Institución Universitaria.

- excesiva carga docente.

- falta de supervisión en el ámbito docente.

- masificación de las aulas, sobre todo en los primeros cursos de carrera.

- pasividad y falta de motivación de una parte del alumnado, así como su bajo nivel de preparación.

Los aspectos positivos más destacados para los profesores contratados son los siguientes: 
- poder realizar el trabajo que les gusta.

- la libertad y flexibilidad que ofrece trabajar como profesor de universidad.

- la posibilidad de realizar la tesis doctoral como primer proyecto de investigación.

Los aspectos positivos más destacados para los profesores fijos son los siguientes:

- poder realizar el trabajo que les gusta.

- libertad y flexibilidad en la organización y realización de su trabajo como características específicas.

- la estabilidad laboral.

- el prestigio y el status que representa ser profesor de universidad.

La posición básica de los profesores contratados se centra en la incertidumbre e inestabilidad laboral. La descripción que hacen de su trayectoria académica está fuertemente impregnada, en la mayoría de los casos, por una serie de expectativas que se van frustrando con el paso del tiempo. Alguno de ellos hablan claramente de "sentirse engañados" al constatar que las promesas que se les hicieron al ingresar en los departamentos no han respondido a la realidad, y siguen estando marcados por la promesa del futuro, que esperan "algún día llegará", pero no saben cuándo ni, en muchos casos, cuál es el camino "idóneo" que han de seguir para su consecución. Esta gran expectativa se deposita en el acceso a una estabilidad laboral.

Por tanto, estos profesores muestran un gran estado de ansiedad, acompañado de una falta de confianza en el futuro debido a la situación real que viven: congelación de plazas nuevas, disminución de recursos económicos y materiales, competitividad entre los compañeros, que produce una posición fuertemente individualista y de desconfianza, cuya traducción anímica consiste en una importante desmoralización profesional y personal, que no se refleja en el plano motivacional/actitudinal.

Estos profesores denuncian que la obtención o no de su ascenso académico no depende de criterios objetivos, sino que depende fundamentalmente de encontrar posiciones de "apadrinamiento". Hablan de la existencia de baremos objetivos, pero resaltan que su importancia es mínima debido a que si no existe alguien que les apoye y les promocione, los criterios objetivos son "papel mojado". Por tanto, manifiestan una posición de indefensión de carácter estructural, que tan sólo se puede paliar mediante las relaciones profesionales, que en realidad se trata de relaciones personales, con profesores que tengan cierto grado de poder.

Además consideran que los problemas laborales con los que se encuentran actualmente se explican en gran medida desde la ausencia de una planificación clara, precisa y transparente de la carrera académica. Asimismo, esta situación se produce por la perversión de gran parte del contenido de la Ley. Este último as- 
pecto también es mencionado por los profesores fijos, pero se analizará con detenimiento más adelante.

Por otro lado, no existen diferencias significativas dentro de la categoría de profesores contratados. Su discurso es homogéneo. Lo que predomina es que este colectivo tiene una clara conciencia de su posicionamiento en el entramado del conjunto del PDI. Dicho posicionamiento, se caracteriza por su precarización laboral y su baja remuneración económica; es decir, se sienten "mano de obra barata", lo que no significa que su nivel de dedicación, traducido en horas de docencia e investigación, sea inferior al de los profesores fijos. Por el contrario, muchos de ellos afirman tener una carga docente muy superior, no sólo a la establecida por su contrato, sino en relación con la que realizan muchos de los profesores fijos. Ahora bien, de todo esto no ha de deducirse una postura de confrontación de los profesores contratados hacia los fijos. Por el contrario, aquellos consideran a éstos como compañeros, que, si bien poseen una situación laboral considerablemente mejor, están afectados por problemas comunes y sienten que entienden y comparten el diagnóstico y las quejas de los contratados.

Los aspectos que los profesores contratados destacan como positivos tienen un peso menor desde el punto de vista cuantitativo, sin embargo, desde una perspectiva cualitativa operan como un gran contrapeso, de forma que les hace seguir en la carrera académica. Sin duda obtienen beneficios, principalmente secundarios, que entran en juego y que compensan todo lo expuesto.

Con respecto a los profesores fijos, éstos tienen valoraciones bastante homogéneas tanto sobre los aspectos positivos como sobre los negativos. Hablan desde una posición claramente consciente de su estabilidad laboral, y desde el sentimiento de pertenencia al estamento del PDI en la Institución Universitaria. Asimismo, manifiestan una conciencia de status y de cierto prestigio, sobre todo los catedráticos. Se sienten miembros "de la casa", pero su discurso mayoritariamente es muy crítico tanto con la organización como con las posibilidades que tienen para el desempeño de sus funciones. Sin embargo, se observa un discurso más sosegado, al menos en el tono y la forma en que realizan las críticas. Pero las diferencias de diagnóstico con respecto a los profesores contratados no son significativas.

Desde este panorama, las responsabilidades que aducen como causantes de esta situación laboral que describen las sitúan básicamente en tres ámbitos:

- Político: consideran que hay una falta de interés real en los responsables políticos por solucionar los problemas que tienen. Una parte piensa que el Gobierno desarrolla estrategias políticas encaminadas a desatender intencionadamente a la Universidad Pública, e ir potenciando el crecimiento de las universidades privadas. Uno de los argumentos utilizados es la creación de un estado de opinión mediático que desacredita a la UPE desde informaciones que consideran falsas. Esto se piensa tanto en las Universidades de Madrid como en las de Barcelona. Asimismo, los entrevistados de Barcelona también son críticos con el Gobierno de Cataluña, ya que con- 
sideran que potencia una única Universidad (la Universidad Pompeu Fabra) en detrimento del resto.

Ahora bien, el posicionamiento ideológico de estos profesores tiende a establecer diferencias entre los planteamientos y las directrices de gobiernos dirigidos por unos partidos políticos u otros. Pero, a pesar de ello, lo dominante es la desconfianza de este ámbito en su conjunto.

- Los rectores y sus equipos de gobierno: Aquí se tiende a reproducir el mismo discurso que respecto al mundo de lo político; pero, se añade un elemento fundamental: la sensación de enorme distancia de los equipos rectorales de los problemas concretos de las facultades pero, sobre todo, de los departamentos, y, en ultima instancia, de los sujetos concretos que trabajan en la Universidad.

- Los problemas estructurales que arrastra la Universidad Pública en España desde hace muchísimo tiempo: Se considera que es un tema tremendamente complejo, donde confluyen muchos intereses, sobre todo de grupos de poder, que lo único que se ha hecho por parte de los diferentes sectores con responsabilidad ha sido "poner paños calientes". Pero no se ha planteado una reforma en profundidad, bien porque no se ha podido, bien porque ha sido obstaculizada por intereses provenientes de esos grupos de poder. Algunos profesores han señalado que sí ha habido intentos de llevar a cabo dicha reforma, pero que la aplicación de la misma ha traído como consecuencia la perversión de los objetivos que buscaba.

Una minoría de entrevistados ha señalado que la responsabilidad de los problemas laborales obedece también al mal funcionamiento de ellos mismos. Este discurso autoinculpatorio se traduce en aspectos tales como los siguientes:

- afán de poder desmesurado de algunos profesores que desatienden sus obligaciones profesionales para dedicarse a tareas "extraacadémicas".

- pasividad en los profesores ante los problemas con que se encuentran.

- falta de imaginación para afrontar dichos problemas.

- falta de conciencia de pertenencia de grupo en la comunidad universitaria para mantener posiciones de reivindicación.

El conjunto de los procesos perceptivo-valorativos de este mundo profesional constata una realidad que va a tener un enorme peso específico en el análisis de los significados y el sentido que posee para este colectivo el concepto de satisfacción laboral:

- los aspectos negativos se caracterizan por referirse mayoritariamente a temas de orden físico-material que corresponden al plano de lo fáctico, de lo concreto, de lo tangible y de lo real.

- los aspectos positivos se refieren de forma mayoritaria a criterios que pertenecen al ámbito de los valores y de las cargas y connotaciones sim- 
bólicas que conlleva el desempeño de este trabajo. Pertenece al plano de las representaciones simbólico-sociales, del imaginario colectivo.

\section{SATISFACCIÓN LABORAL: CAMPOS SEMÁNTICOS QUE CONSTRUYEN EL CONCEPTO}

La satisfacción es un concepto que se define tanto por criterios racionales como emocionales. Por tanto, la construcción que se realiza de este concepto está atravesada por parámetros interrelacionados desde la razón y desde la emoción. Desde determinados modelos teóricos provenientes del campo de la psicología ${ }^{1}$, se mantiene que el deseo es un elemento fundamental en todo proceso de motivación. Los resultados de las acciones motivadas desde el deseo constituyen una fuente de satisfacción en el ser humano. No obstante, desde esta perspectiva, el deseo es inalcanzable y por tanto actúa como motor de las acciones de las personas.

La explicación de este aspecto desborda los objetivos del estudio, pero es necesario tenerlo presente para una comprensión más completa de la trama que conforma la satisfacción humana en general y la satisfacción laboral.

No es posible entender la satisfacción laboral solamente desde criterios ambientales, exógenos al sujeto, entendido éste en su vertiente psíquica, sin duda fundamentales. También deben considerarse los aspectos no racionales, no conscientes, latentes que este análisis permite hacer emerger y poner de manifiesto. De esta forma puede contemplarse la realidad analizada desde una posición más holística.

En la construcción de este concepto, los profesores unas veces se han referido a él de forma espontánea y otras de forma sugerida. Pero durante prácticamente todo el discurso está presente, de manera latente o patente, el concepto de satisfacción.

En su análisis se han utilizado distintos enfoques pertenecientes a diversos modelos teóricos y a distintas disciplinas, fronterizas entre sí. De este modo, las perspectivas de análisis se abordan desde: a) la sociología, para ver las estructuras y los procesos colectivos que están operando en el conjunto de la sociedad y su incidencia en el mundo universitario; b) la psicología social, para analizar los funcionamientos de grupos, formales e informales, de profesores y las interrelaciones entre ellos; c) la psicología, para conocer los procesos cognitivos, actitudinales y valorativos de estos sujetos; d) la semiótica, para analizar el contenido de los discursos, su sintaxis y cómo expresan sus distintos niveles de habla con el fin de poner en relación el texto, discurso producido, con el contexto, posición estructural a la que pertenece el sujeto que habla; $y$, por último, e) la an-

${ }^{1}$ En concreto, los que defienden una visión de los procesos de la psique humana desde una perspectiva dinámica a partir de las aportaciones de los enfoques de las distintas escuelas Psicoanalíticas

EMPIRIA. Revista de Metodología de Ciencias Sociales. N. ${ }^{\circ} 11$, enero-junio, 2006, pp. 175-201. ISSN: $1139-5737$ 
tropología estructural, para entender la relación entre los procesos culturales hegemónicos que cristalizan en la forma de interpretar y de actuar en y con la realidad de esta sociedad, y la articulación de estos procesos en las posiciones que mantienen los sujetos entrevistados.

La satisfacción laboral surge asociada a dos aspectos: a) representa un estado de ánimo, plano emocional; b) representa la consecución de unos determinados objetivos, plano racional.

Ambos planos están íntimamente relacionados de forma que no es posible desligarlos, tampoco tendría sentido hacerlo, ya que falsearía la realidad al no tratarse de que el todo sea la suma de las partes (véase Fig.2.).

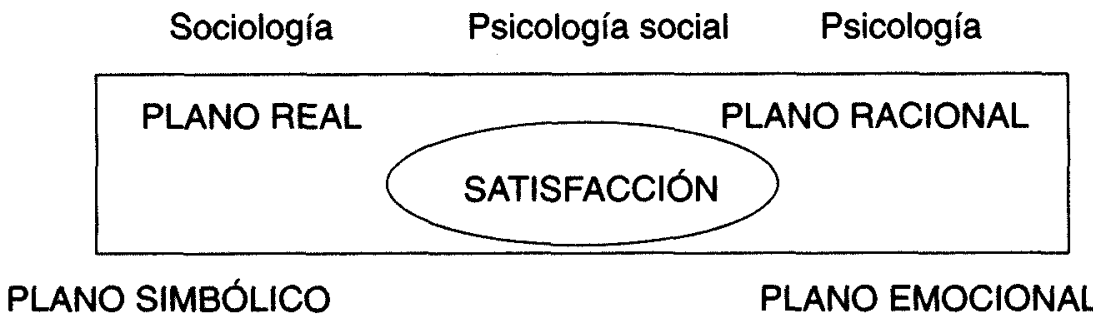

\section{Antropología estructural Semiótica}

Fig. 2: Campos semánticos que constituyen el concepto de satisfacción para el profesorado universitario

La producción discursiva en los campos semánticos que se abren sobre este concepto se ha analizado desde el eje sintagmático, eje horizontal del plano denotativo, y el paradigmático, eje vertical de la cadena asociativa en el plano connotativo. De esta manera, se accede tanto a los distintos significados que tiene la satisfacción laboral como a las distintas áreas que lo integran.

Debe señalarse que los profesores en su conjunto reproducen, un aspecto nuclear, característico de la modernidad o, tardo-modernidad, que es la fuerte impregnación que adquiere lo simbólico, incluyendo en este concepto los índices, iconos y símbolos, según el esquema del semiótico Peirce, en detrimento de lo real. Aquí se quiere poner de manifiesto que la sociedad actual se caracteriza por la prioridad casi absoluta de los significantes y la tendencia a la desaparición de sus significados. Es decir, los individuos perciben, valoran y se "mueven" en el mundo de las representaciones simbólicas y se alejan cada vez más de aquellos aspectos de la realidad a la que hacen referencia esas representaciones. Por tanto, lo que predomina como paradigma social es el mundo de la imagen, de los atributos y valores, que constituyen las representaciones sociales dominantes que hegemonizan y legitiman los estados de opinión. Por ello, tienen cada vez más dificultad no sólo para relacionarse sino, para acceder y conocer el mundo de lo real. En definitiva, los referentes simbólicos se independizan de lo referido 
(mundo de lo real) y adquieren autonomía propia. En este sentido, se están produciendo importantes transformaciones en los procesos cognitivos, valorativos y actitudinales de los individuos.

De esta forma, la satisfacción laboral es un concepto que se construye en muchos momentos desde un plano ideal $\mathrm{y}$, al no ser posible como realidad fáctica, genera insatisfacción y a veces frustración.

Este aspecto es importante porque opera entre los profesores con distinta intensidad y es un elemento que está presente a la hora de entender el alto grado de insatisfacción que manifiestan la mayoría de ellos.

Las vivencias de estos profesores se sitúan básicamente, en el pasado o en las expectativas del futuro, pero apenas aparece en sus discursos la referencia al presente como momento experiencial que tenga valor en sí mismo. Esto se relaciona directamente con lo anterior, o es consecuencia de ello. La persona que siente, piensa y vive dentro de los imaginarios, no encuentra valor ni gratificación en su experiencia del presente, porque éste siempre tiene un déficit de imperfección, de no adecuarse a "lo que debería ser". Esta es una de las características que define la sociedad actual, la evacuación del presente como tiempo experimentado.

Por tanto, la satisfacción, laboral o de otra índole, es un proceso que tiende al infinito, ya que el individuo nunca llega a sentirse satisfecho, por cuanto su consecución, desde la perspectiva expuesta, implicaría acceder por completo a la "idealidad".

Los campos de significación fundamentales que tiene la satisfacción laboral para el conjunto de los profesores entrevistados son los siguientes:

- La dedicación a una profesión que es la que más gusta. La palabra vocación está muy presente en la mayoría de los profesores, tan sólo no aparece en los que pertenecen a las escuelas y facultades técnicas.

- El reconocimiento profesional y personal, de los compañeros, de la Institución Universitaria a la que pertenecen, del mundo universitario en su conjunto, y de la sociedad. Este reconocimiento opera en distintos niveles: económico, valoración de la aportación científica e intelectual, capacidad docente e investigadora y obtención de prestigio y status, constituidos a partir de sus conocimientos y de su acceso al "Saber", de la aportación de dichos conocimientos mediante la docencia y, sobre todo, las publicaciones, y por la pertenencia a este colectivo universitario y la categoría conseguida: la figura de catedrático como máximo exponente de la carrera académica.

- La valoración enormemente positiva de las características que tiene la realización de este trabajo: su libertad y flexibilidad para organizarse el desempeño de sus tareas. Este aspecto marca una gran diferencia cuando surge la comparación con otros trabajos.

- La posición de estabilidad laboral que conlleva ser profesor fijo: genera tranquilidad en los que ya son funcionarios y deseo y expectativas de conseguir una plaza fija en los profesores contratados (véase Fig.3). 


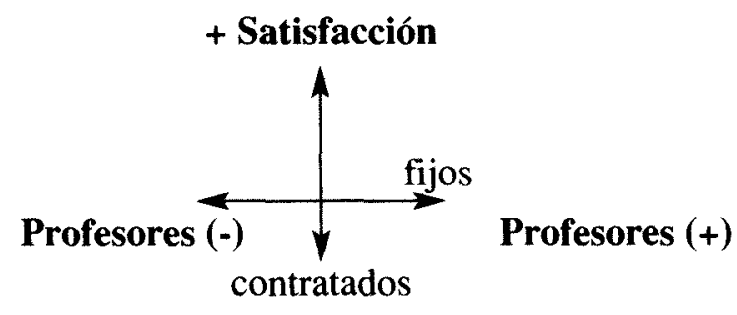

\section{- Satisfacción}

Fig. 3: Nivel de satisfacción según la figura del profesorado

La satisfacción laboral adquiere su máximo sentido entendida como satisfacción personal. En esta profesión, percibida como un proceso en el que está involucrada la persona íntegramente, el avance en el ascenso de la carrera académica se siente como un avance y un crecimiento en el plano personal.

Los profesores evidencian a través de sus discursos la apuesta personal que les supone la consecución de los objetivos laborales y la obtención de "las metas" que se proponen. De manera que los éxitos y los fracasos profesionales son vividos como personales. El grado de implicación que representa el trabajo de profesor universitario desborda las fronteras de lo que habitualmente delimita el ámbito laboral, lo transciende y sus resultados se valoran desde posiciones magnificadoras.

Esta situación produce una intensa identificación entre los logros y los fracasos profesionales y los personales. Los discursos manifiestan, en momentos de gran condensación de sentido, que la situación profesional de los profesores y sus expectativas inciden de manera profunda en su autovaloración personal e incluso en sus procesos de identidad.

El desempeño de esta labor no sólo se ubica en la esfera de lo público sino que también está afectando el ámbito privado. Ya se ha señalado más arriba que "esto es más que un trabajo", "es un estilo de vida", incluso algunos llegan a afirmar que "es el propio sentido de mi vida el que está en juego".

No es casual que "lo vocacional" se instale rápidamente en el discurso de la mayoría de los individuos Surge de manera espontánea y opera como elemento de aglutinación de muchos factores, ya que se utiliza como motor de un amplio despliegue de significados, como los siguientes:

- Llamada a la realización de una profesión que tiene sus raíces en el interior del sujeto.

- Aptitudes personales para el buen hacer de las tareas propias del profesor universitario.

- Ejercicio de una profesión, cuya motivación principal no es de índole económica, sino de aportación al mundo cultural y científico y, en última instancia, a la sociedad. 
- Conceptualización de esta profesión como algo más que "un simple trabajo", un espacio de realización personal.

- Carta de presentación que opera como justificación y cobertura de una posición que manifiesta fuerte insatisfacción, sobre todo en los profesores contratados.

La satisfacción laboral, como concepto construido por los propios profesores entrevistados, se sustenta en dos ejes básicos:

- Eje de los elementos que integran el mundo simbólico: la imagen de lo que representa la Universidad y ser profesor universitario, el status que comporta ejercer esta profesión y obtener una categoría profesional, el prestigio avalado por cumplir una función social dotada de valor, la distinción de ejercer una profesión al margen del mercado (el profesor universitario no siente que tenga que entrar en el juego de la oferta y la demanda), etc.

- Eje de los elementos físico-materiales y económicos: obtención de una remuneración económica "digna", disponibilidad de medios y recursos para ejercer con criterios de calidad la docencia y la investigación, acceso a medios para relacionarse con universidades extranjeras (posibilidad de viajes a congresos, cursos, estancias, etc.) que permita estar en la punta de lanza de los trabajos reconocidos por la comunidad científica internacional, etc.

Ambos ejes están íntimamente relacionados sin embargo, lo simbólico opera con mayor determinación y con un mayor peso específico. De hecho, el poder que detentan determinadas figuras pertenecientes a la Institución Universitaria es fundamentalmente de carácter simbólico. Es evidente que ello tiene su refrendo en aspectos económicos y materiales, sin los cuales no se mantendría dicho poder. Por tanto, ambos aspectos: forman una realidad indisoluble, pero con características diferenciales.

Es importante detenerse en un aspecto concreto que permite comprender cómo funciona la satisfacción en este ámbito laboral. Los aspectos relacionados con el eje de lo simbólico se constituyen, sobre todo, a partir del valor asignado al mundo universitario y a la Institución en particular. Los profesores universitarios sienten que es la Institución, sus valores y atributos, la que les inviste de los mismos. Esto no implica que se produzca una autoanulación valorativa. Sin embargo, pone en evidencia que la construcción de valor que conforma la imagen de su profesión, y, por tanto, el concepto de satisfacción referida a la misma, proviene de forma prioritaria y muy potente del valor asignado a la Institución.

Como se ha mencionado anteriormente, se producen posiciones diferenciales sobre la articulación y la plasmación en la realidad concreta y cotidiana de la aplicación de los criterios que definen la satisfacción. Los profesores contratados centran gran parte de la satisfacción en la obtención de una estabilidad laboral y en una mejora de sus condiciones económicas, mientras que los profesores fijos ponen el énfasis en la obtención de recursos y medios materiales y humanos para

EMPIRIA. Revista de Metodología de Ciencias Sociales. N. ${ }^{\circ} 11$, enero-junio, 2006, pp. 175-201.

ISSN: $1139-5737$ 
la realización de las tareas docente e investigadora y reclaman una disminución de los trámites burocráticos en la gestión de proyectos.

Otro aspecto relevante en la construcción del concepto de satisfacción de los profesores es el binomio docencia-investigación. Unos consideran que es prioritaria la docencia, otros piensan que es la investigación y otros valoran ambas tareas como complementarias. Las diferencias valorativas no guardan relación con las variables seleccionadas, sino que responden a criterios personales. Sí acaso, existe cierta tendencia de los profesores pertenecientes a las áreas de Ciencias Experimentales y de la Salud y de las Escuelas Politécnicas a valorar más la investigación como criterio motivacional, y los pertenecientes al área de Humanidades a mostrarse más motivados por la docencia, manteniéndose los pertenecientes al área de Ciencias Sociales y Jurídicas en una posición de equilibrio con respecto a ambas tareas. Pero, de cualquier modo, se considera que tanto la docencia como la investigación hay que realizarlas con criterios de calidad.

Por tanto, ambas funciones son vistas como elementos fundamentales que deben integrarse dentro de los indicadores de satisfacción (véase Fig.4.).

- ENSEÑANZAS TÉCNICAS

- CIENCIAS EXPERIMENTALES Y DE LA SALUD

- CIENCIAS SOCIALES Y JURÍDICAS

- HUMANIDADES

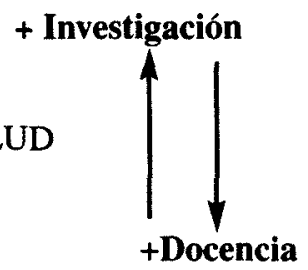

Fig.4: Actitud ante la investigación y la docencia según área de conocimiento

La consideración acerca de si la carga docente debe ser menor para tener más tiempo disponible para la investigación, es defendida por los que priorizan a esta última, y la defensa de la docencia, tanto en su dedicación como su nivel de calidad, dentro de los criterios curriculares, es mantenida por los que se sienten más motivados y dan más importancia a la docencia. Resulta llamativo que se hable de "carga" docente y de "actividad" investigadora. Parece que se denomina a cada una de estas tareas según el peso que tienen una y otra y, el valor que se les otorga.

Sobre este tema hay un comentario que se hace presente en la mayoría de las entrevistas y grupos de discusión, y es que la Ley prima la investigación, y más que la investigación la publicación, como único criterio curricular, en detrimento de la docencia. Como consecuencia de ello se percibe una falta absoluta de interés por evaluar el nivel de docencia de los profesores, y su nula incidencia en la promoción de la carrera universitaria. Esto se contrarresta con la actitud vocacional para el ejercicio de esta tarea, y por la gratificación que aporta al sujeto la relación con el alumnado. Ello a pesar de las críticas que surgen acerca de las características actuales de dicho alumnado, que lo consideran falto de for- 
mación, a veces con escaso interés por aprender y sólo motivado por la obtención del título con el mejor expediente académico posible. De manera que muchos alumnos convierten su paso por la Universidad en un trámite para obtener una titulación que tenga un valor de cambio en el mercado laboral, desinteresándose por considerar este espacio como un lugar de aprendizaje y de formación.

Por último, otro aspecto fundamental en la configuración de los parámetros de satisfacción son las relaciones profesionales y personales entre compañeros. Se considera que es fundamental tener un clima de trabajo agradable, donde pueda existir el compañerismo y el trabajo en equipo. Además, los profesores contratados señalan la importancia que tiene para ellos el apoyo del departamento y de "sus jefes" para conseguir una estabilidad laboral.

Por consiguiente, los elementos que integran este concepto están delimitados por dos ejes estructurantes:

- Eje horizontal: integra los elementos que tienen que ver con el desempeño de tareas y funciones, y las áreas que lo rodean. Se refiere fundamentalmente a los ámbitos de actuación del sujeto en los que existe una cierta autonomía de decisión y funcionamiento.

- Eje vertical: está constituido básicamente por los aspectos relacionados con la organización y la Institución. Se refiere principalmente a elementos de carácter estructural y tiene relación con aquellos elementos que son heterónomos al sujeto.

En definitiva, los campos de significación que conforman la satisfacción laboral están definidos por pares semánticos, como los siguientes:

- Satisfacción laboral vs. satisfacción personal.

- Mundo de lo simbólico (representaciones e imaginario individual y colectivo) vs. mundo de lo concreto (aspectos económicos y materiales): plano ideal vs. plano de lo real.

- Ámbito público vs. ámbito privado.

- Profesores fijos vs. profesores contratados.

- Profesión-vocación vs. trabajo.

- Docencia vs. investigación.

- Profesor vs. alumno.

- Plano de la Institución vs. plano del individuo.

- Sociedad vs. Universidad.

Atendiendo a las variables en torno a las que se han estructurado los resultados de este estudio, la más discriminante es el tipo de vinculación contractual con la institución universitaria, ser profesor fijo o contratado. De acuerdo a esta variable se construye todo el entramado de satisfacción-insatisfacción. 
La variable género ofrece matices diferenciales en los posicionamientos de las profesoras y de los profesores. Dichas diferencias se centran fundamentalmente en los siguientes aspectos:

- Las profesoras ejercen una posición crítica sobre el nivel de satisfacción laboral de igual modo que lo hacen los hombres. Sin embargo, sus aspiraciones en la carrera académica están condicionadas por hacerla compatible con su mundo privado/personal/familiar. Valoran su profesión, pero la mayoría considera que su importancia esta delimitada por el ámbito personal.

- Los profesores, por el contrario, se vuelcan mucho más en la carrera académica, sin las limitaciones que aducen las mujeres, debido a que, tradicionalmente, determinadas responsabilidades familiares les corresponden a ellas. Este aspecto no aparece de forma explícita en sus discursos, bien porque no son plenamente conscientes, bien porque sería "políticamente incorrecto". Sin embargo se detecta en el análisis de sus aspiraciones en la carrera académica, como se mencionó anteriormente.

Se han detectado otras dos variables discriminantes no contempladas en la muestra, que operan en la percepción y valoración de la satisfacción laboral, el ciclo vital de los profesores y la posición ideológica. Respecto a la primera:

- Los profesores de mayor edad que son fijos y gozan de estabilidad laboral, adoptan posiciones más desapasionadas y escépticas, a la vez que muestran poca confianza en cuanto a la existencia de soluciones que puedan mejorar su situación laboral. No obstante, mantienen una posición crítica frente a la situación de los profesores contratados.

- Los más jóvenes mantienen posiciones actitudinales y motivacionales más activas, acordes tanto con la etapa de su ciclo vital como de su ciclo profesional.

Respecto a la posición ideológica se destaca que:

- los sujetos que mantienen criterios ideológicos próximos a lo que tradicionalmente se ha entendido como izquierda adoptan una postura más crítica con su situación laboral y con el funcionamiento actual de la Universidad, centrando la responsabilidad de ello fundamentalmente en la esfera política.

- Los sujetos que manifiestan una ideología próxima al centro-derecha, tienden a hacer un diagnóstico, en el que hablan de una mala situación laboral de los profesores de la Universidad Pública Española. 


\section{CONCLUSIONES}

Para los profesores del la Universidad Pública Española, lo vocacional está muy presente como concepto. La "satisfacción" significa "la dedicación a la profesión que más les gusta y el reconocimiento profesional y personal de los compañeros, de la Institución Universitaria y de la sociedad en su conjunto.

Por tanto la satisfacción laboral tiene campos de significación concretos, se hace una valoración enormemente positiva, de la libertad y flexibilidad para organizar el desempeño de sus tareas y la estabilidad laboral que conlleva ser profesor fijo como características intrínsecas de su trabajo, que está asociado con el gusto, el placer, la elección, el deseo, etc. Pero cuando se menciona este concepto se produce una indiferenciación entre lo que representa o sienten como satisfacción personal y satisfacción laboral.

Por tanto, la satisfacción laboral adquiere su máximo sentido entendida a través de la satisfacción personal. El profesor de universidad se aplica y se implica en el desempeño de su profesión, de manera, que sus éxitos y sus fracasos profesionales se viven como éxitos y fracasos personales.

Ahora bien, la satisfacción o insatisfacción laboral está determinada por el tipo de vinculación contractual con la institución universitaria, ser profesor contratado o profesor fijo. Esta variable configura la base sobre la que se articula todo el entramado de satisfacciones, insatisfacciones, expectativas y frustraciones.

El paso de profesor contratado a profesor fijo genera un ámbito que se encuentra determinado por el itinerario que han de seguir y éste es un factor de profunda insatisfacción. Insatisfacción por la incertidumbre sobre: su futuro laboral en la Universidad, el tiempo que van a tardar en poder obtener una estabilidad laboral, la precariedad económica, por la dependencia para su ascenso en el mundo académico de las relaciones profesionales y personales que mantienen con sus compañeros que ocupan cargos de jerarquía superior, ya que los baremos objetivos por sí mismos sienten que no son suficientes, y necesitan el aval y el apoyo de los profesores con capacidad para promover este ascenso.

Sin embargo, todo esto se soporta por la promesa de futuro, que conlleva: la estabilidad laboral, sueldo más digno, libertad y flexibilidad para organizar el desempeño de sus funciones laborales, prestigio y status.

Una vez que se alcanza la meta de «ser profesor fijo», la primera etapa, está caracterizada por el disfrute. Pero con el transcurso del tiempo, el profesorado fijo muestra un sentimiento de falta de reconocimiento y falta de pertenencia institucional que se traduce en ausencia de reconocimiento de su labor, de su "prestigio" y de falta de reconocimiento social. Por tanto, muestran un grado de insatisfacción laboral significativo, sumado a la falta de medios y recursos económicos, materiales y humanos, para realizar con criterios de calidad las tareas docentes y fundamentalmente de investigación.

En el binomio docencia-investigación se valora que es la investigación, y sobre todo su publicación, la que realmente tiene importancia porque es la única 
que repercute curricularmente. Sin embargo, se considera que la docencia apenas se tiene en cuenta, y se demanda que existan mecanismos de evaluación eficaces que redunden en la obtención de beneficios concretos.

Por último, hay que señalar que este colectivo reclama una revisión de la situación existente con el fin de adaptar todos los criterios de valoración y evaluación en los diferentes ámbitos a la situación real actual. 


\section{BIBLIOGRAFÍA}

ÁlvAREZ, J. M. ((2000)) Dialéctica, currículum y evaluación. Niño y Dávila.

ÁLVAREZ, J. M. (2001) Evaluar para conocer, examinar para excluir Morata.

ÁlvAREZ, M. (1998): El liderazgo de la calidad Total Madrid, Escuela Española.

ÁlvAREZ, V. y LÁzARo, A. (Coord.) (2002) Calidad de las universidades y orientación universitaria. Málaga. Ediciones Aljibe.

Álvarez, V. (Dir.) (2000): Propuestas del profesorado bien evaluado para potenciar el aprendizaje de los estudiantes. Universidad De Sevilla.

Almarcha, A.; GonZÁlez, B. y GonZÁlez, C. (1994). «Cambio y desigualdad en el profesorado universitario». Revista Española de Investigaciones Sociológicas, 66. Ed. CIS. Madrid.

ARBIZu, F. (1994). La Función docente del profesor universitario. Bilbao: Servicio Editorial de la Universidad del País Vasco.

BARTHES, R. (1971) Elementos de semiología. Madrid, Alberto Corazón. Benedito, V. (coord.), (1992). La formación del profesorado universitario. Madrid: MEC

BARO, E. et al. (2000). Informe Universidad 2000. Conferencia de Rectores Universitarios Españoles. Informe de la CRUE. Madrid.

BOURDIEU, P. (1985) ¿Qué significa hablar? Madrid, Akal.

CABALlERo MARTINEZ, J. et al. (1993). La satisfacción del profesorado universitario informe de una investigación realizada en la Universidad de Granada. Universidad de Granada. Servicio de publicaciones.

CASTELLS, M. (1999) La era de la información: economía sociedad y cultura. Madrid, Alianza ( $1^{a}$ ed. 1997)

CluB GESTIÓN DE CALIDAD (1998). Mejora en la formación universitaria: sugerencias desde la empresa. Ed. Club Gestión de Calidad. Madrid.

CURIER, J. y NEWSON, J. (1998). Universities and globalization: critical perspectives. Ed. Curier J. Newson J. Thousand California.

Chomsкy, N. (2000) Chomsky on MisEducation. Ed. Rowman \& Litlefield. New York.

FERNÁNDEZ ENGUITA, M. (1993). «La profesión frente a su público». El País. 30 de marzo.

Finkelstein, M. J.; SeAl, R. K. y Schuster, J. H. (1998). The New Academic Generation. The Johns Hopkins University Press, Baltimore.

Frías, R. (1994). El stress en las UCI Ed. CDN y UAH. Madrid

FUNDACIÓN SANTILlaNA (2002). Aprender para el futuro. Universidad y sociedad. F. Santillana. Madrid.

GarCÍA FerRando, M. y LóPEZ-ARAguren, E. (1991). «Experiencia de investigación social en la universidad española». Revista Española de Investigaciones Sociológicas, 56. Madrid.

GINER DE Los Rfos, F. (1990). Escritos sobre la universidad española. Ed. Espasa Calpe. Madrid.

GIDENS, A. (2000). Un mundo desbocado. Los efectos de la globalización en nuestras vidas. Ed. Taurus. ( $1^{\mathrm{a}}$ ed. Londres 1999). Madrid.

GrEMAS, J.A. (1973) En torno al sentido. Madrid, Fragua.

IвÁÑEZ, J. (1979) Más allá de la sociología. El grupo de discusión: Técnica y crítica. Madrid, Siglo XXI. 
LÁZARO LORENTE, L. M. (1993). Congreso Internacional sobre formación pedagógica del profesorado universitario y calidad de la educación. Universitat, Servei de formació permanent: C.I.D.E., D.L. Valencia.

LÉvi-Straus, C. (1977) Antropología estructural. Buenos Aires, Universitaria B.A. (1. ed. París 1958).

MARTÍN GARCía, V. (1999). «La evaluación universitaria: paso obligado de la calidad educativa». Sociedad y Utopía. Madrid.

MARTínez G. TeBlas, A. (2000). Economía política de la globalización. Madrid.

MAYOR ZARAGOZA, F. (1996). «La educación superior ante los retos del siglo XXI». Discurso de la inauguración del año académico. UPM. Madrid.

De Miguel, J. M. et al. (2001). Excelencia. Calidad de las universidades españolas. Ed. CIS. Madrid.

MichaVILA, F. y Calvo, B. (1998). La Universidad española hoy. Ed. Síntesis. Madrid.

MORA, J. G. (2000). El profesorado universitario: situación en España y tendencias internacionales. Consejo de Universidades. Madrid.

ORTi, A. (1989) «La apertura del enfoque cualitativo o estructural. La entrevista abierta y la discusión de grupo» en García Ferrando, M. et al. El análisis de la realidad social, Madrid, Alianza.

PAUL, K. (1999). «University rankings: How helpful are they to you?» Sympatico University Bound. [www.sk.sympatico.ca...Bound/article_univrsity_rankings.html, 29 julio]

SÁNCHEZ, F. J. et al. (1986). Enseñanza universitaria y mercado de trabajo: el primer empleo de los titulados universitarios. Ed. Fundación universidad empresa. Madrid.

SÁnCHEZ-Ferrer, L. (1996). Políticas de reforma universitaria en España 1983-1993. Fundación Juan March. Madrid.

SELINGO, E. (2000). «How many research universities does a State needs». The Chronicle of Higher Education. [http://chronicle.com/colloqui, 11 de febrero]

SCHARGEL, F. P. (1997). Cómo transformar la educación a través de la gestión de la calidad total. Ibd. Limited. January.

TENA ARTIGAS, J.; CORDERo, L. y Díaz JARES, J. L. (1976). La universidad española: datos para un problema. Confederación Española de Cajas de Ahorros. Madrid.

TORTELLA, G. (1999). «La Universidad no va bien». El País, 21 de junio.

Velasco, C. y Velásquez, M. P. (1983). "Notas y disfunciones en la distribución del profesorado universitario en España: una aproximación". Revista Española de Investigaciones Sociológicas. Madrid.

ZuBIETA, J. C. et al. (1992). Satisfacción e insatisfacción de los enseñantes. MEC. 


\section{RESUMEN}

Los campos de significación fundamentales que tiene la satisfacción laboral son: la dedicación a la profesión que más gusta, reconocimiento profesional y personal, libertad y flexibilidad para organizarse el trabajo y la estabilidad laboral.

La satisfacción laboral adquiere su máximo sentido entendida a través de la satisfacción personal.

La satisfacción o insatisfacción, están determinadas por el tipo de relación contractual (fijo o contratado) con Institución Universitaria.

\section{PALABRAS CLAVE}

Satisfacción, profesorado universitario, libertad, flexibilidad, docencia, investigación, recursos, estabilidad.

\section{ABSTRACT}

The main categories for the measurement of job satisfaction were: job fulfillment, professional and personal appreciation, the possibility for flexible work schedule job stability. Thus, job satisfaction is positively correlated with personal satisfaction.

Either satisfaction or insatisfaction relys on the type of contract and conditions agreed with the Institution.

\section{KEY WORDS:}

Job satisfaction, teaching staff, freedom, flexibility, teaching, research, resources, job stability. 